\title{
LA ESCUELA EN JUEGO
}

\author{
Aldo Román Césaro \\ Nicolás Viñes \\ Universidad Nacional de la Plata (Argentina)
}

RESUMEN: La extensión universitaria es entendida en el sistema universitario argentino como uno de los tres pilares fundamentales que lo componen, junto con la enseñanza y la investigación. En este marco, las prácticas de extensión son experiencias de intervención social, que ponen a prueba los saberes adquiridos en la formación de grado, saberes que se deconstruyen y reconstruyen a partir en una constante interacción entre los diversos actores sociales.

El proyecto de extensión que describimos en el siguiente artículo surgió a partir de algunas observaciones focalizadas en la comunidad y la escuela de inserción. En ese diagnóstico inicial descubrimos que las prácticas lúdicas en niños y adolescentes del barrio en cuestión, se encontraban condicionadas por dos factores importantes: uno de índole social y el otro por la falta de disponibilidad de espacios para jugar. Por tal motivo creíamos relevante la recuperación de la práctica de algunos juegos desde la escuela, entendiéndola como un "territorio" socialmente legitimado, pero además como un lugar posible para generar políticas educativas hacia la transformación, entendiendo que tal como estaban las cosas era necesaria una reapropiación del juego como derecho y necesidad.

ABSTRACT: The university extension, it is understood in the Argentine university system as one of the three pillars that comprise it, along with teaching and research. In this framework, extension practices are experiences of social intervention, which test the skills acquired in undergraduate training, skills that are deconstructed and reconstructed from a constant interaction between the various social actors.

The extension project, described in the following article came from some observations focused on community and school integration. In this initial analysis found that leisure practices in children and adolescents in the neighborhood in question, were conditioned by two factors: one social and the other by the lack of availability of space for playing. For this reason we thought relevant to recover 
the practice of some games from the school, understanding it as a "territory" socially legitimized, but also as a possible place to generate educational policies towards transformation, understanding that as things stood there was a need reappropriation of the game as a right and need.

PALABRAS CLAVE: Extensión universitaria-juego y jugar-intervención sociocomunitaria.

KEYWORDS: University Extension-game and play-Community social intervention.

\section{Presentación}

La extensión universitaria es entendida en el sistema universitario argentino como uno de los tres pilares fundamentales que lo componen, junto con la enseñanza y la investigación. De la investigación surgen los nuevos conocimientos que nutren a la enseñanza, aportando nuevos saberes a estudiantes y docentes, "[...] éstos aportan a la sociedad las herramientas con las que cuenta la Universidad, y las problemáticas en ella encontradas recrean y generan las nuevas líneas de investigación"1.

En este marco, las prácticas de extensión son experiencias de intervención social, que ponen a prueba los saberes adquiridos en la formación de grado, saberes que se deconstruyen y reconstruyen a partir en una constante interacción entre los diversos actores sociales ${ }^{2}$.

Si bien es cierto que no hay práctica social que no sea política, pensar las prácticas de extensión en estos términos implica pensarlas de un modo diferente a como habitualmente se ha hecho. Tradicionalmente se ha concebido a la Extensión Universitaria como una "transferencia de conocimientos" desde los ámbitos académicos productores de saberes a los ámbitos sociales populares, que actúan de manera pasiva como receptores de saberes externos a ellos. Poder pensar las prácticas de extensión como la posibilidad concreta sé hacer "prácticas" las prácticas académicas (sean estas, prácticas-prácticas ó prácticas-teóricas); es decir, Ilevarlas a los contextos sociales generalmente excluidos de los contextos de producción de los saberes y a partir de esas mixturas, relaciones, interrelaciones, idas y vueltas, construir nuevos sentidos y significados sobre las propias prácticas entre los extensionistas universitarios y los receptores sociales de la extensión, esta perspectiva supone un giro en cuanto a la concepción de la extensión.

1. Secretaria de Extensión Universitaria, Universidad Nacional de La Plata, (2007). Bases para la convocatoria de proyectos de Extensión Universitaria.

2. La idea de la deconstrucción de los saberes hace referencia a la posibilidad y necesidad de poner en tensión los saberes adquiridos en la formación inicial; permitiendo, en caso de ser pertinente, discutir con éstos, modificarlos y volver a construirlos. La nueva construcción de los saberes está dada en función de un entrecruzamiento entre los saberes teóricos que circulan en el campo, y que de un modo u otro nos hemos ido apropiando, y las prácticas concretas, esas que suceden cotidianamente y de las cuales las teorías muchas veces no dan cuenta. La deconstrucción y reconstrucción es como desandar el camino andado para volver a andarlo, pero de otro modo. 
La experiencia de extensión universitaria que se presenta en este artículo, está enmarcada en una convocatoria interna para proyectos de Extensión Universitaria de la Facultad de Humanidades y Ciencias de la Educación, de la Universidad Nacional de La Plata, Argentina, en el año 2006, cuyo eje central fue la educación popular, desarrollado en el año 2007. La ejecución y puesta en práctica del mismo durante los años 2008 y 2009, fue en base a convocatorias de la Secretaría de Políticas Universitarias del Ministerio de Educación de la Nación, en el marco del Programa del Voluntariado Universitario.

Para una mejor y mayor compresión del fundamento, puesta en práctica y avance y logros a la fecha, el artículo se estructurará en dos grandes apartados. Inicialmente, se presentará el proyecto original, obviando detalles formales que no son relevantes en este caso, para luego dar cuenta de lo actuado hasta el momento.

\section{EXPERIENCIA DE EXTENSIÓN UNIVERSITARIA}

En el diagnóstico inicial del proyecto observamos que las prácticas lúdicas en niños y adolescentes del barrio en que queríamos llevar a cabo nuestra propuesta de extensión, se encontraban condicionadas por dos factores importantes: cuestiones de índole social y la falta de disponibilidad de espacios para jugar. Por tal motivo creíamos relevante la recuperación de la práctica del juego desde la escuela, entendiéndola como un "territorio" socialmente legitimado, pero además como un lugar posible para generar políticas educativas hacia la transformación, entendiendo que tal como estaban las cosas era necesaria una reapropiación del juego como derecho y necesidad.

Entendíamos que el uso de estos espacios, signados cotidianamente con una carga simbólica orientada hacia el trabajo escolar, podían ser re-significados a partir de las prácticas lúdicas que en ellos se construyeran. Esta nueva asignación de sentidos estaría dada fundamentalmente por la elección de parte de los niños y jóvenes de "entrar a la escuela de un modo no habitual", vinculado sobre todo al sentido estrictamente pedagógico. Creíamos y creemos que mediante el juego, se accede a un doble uso de la libertad, el permiso para jugar y la posibilidad de salir cuando el jugador lo desee, características que la lógica del juego escolarizado no permiten. En síntesis, la escuela es libremente elegida como un lugar de juego.

Por las razones expuestas, presentamos las características generales en las que se enmarcaba la propuesta.

A) La misma fue estructurada a partir de 4 ejes temáticos, que se constituirán en talleres rotativos no simultáneos.

A.1) Taller de juegos tradicionales.

A.2) Taller de fabricación de juguetes.

A.3) Taller de juegos indígenas.

A.4) Taller de juegos alternativos y con materiales no convencionales.

B) Cada uno de estos talleres, estaba atravesado por ejes transversales: acción religante, creatividad, memoria y pasado, exploración y descubrimiento.

C) El espacio a utilizar era el patio de la escuela y algunas aulas. 


\section{Los Destinatarios, ReleVANCIA y JUSTIFICACIÓN DEL PROYeCto}

Serían destinatarios directos del proyecto de treinta a cincuenta niños y jóvenes de entre 6 y 15 años de edad, provenientes de familias de bajos recursos de la zona de influencia de la escuela, aunque no en todos los casos; los mismos están escolarizados.

Los destinatarios indirectos serían los propios graduados en etapa de formación en extensión y estudiantes en iniciación, en ambos casos, de la carrera de Educación Física de la Facultad de Humanidades y Ciencias de la Educación. Universidad Nacional de La Plata.

El lugar de realización fue la Escuela Primaria Básica (EPB) № 62 "República de Chile" y Escuela Secundaria Básica (ESB) № 14, ambas del Barrio Altos de San Lorenzo, de la ciudad de La Plata, Buenos Aires, Argentina.

Tal como explicábamos anteriormente, la justificación del presente proyecto se basó en tres ejes principales.

1) Los jóvenes y niños no tienen la posibilidad de jugar porque sus condiciones socioeconómicas los obligan a realizar tareas laborales, o muchas veces a asumir el cuidado de familiares cercanos (hermanos menores, sobrinos, etc.).

2) Quienes tienen la posibilidad de hacerlo, no tienen acceso a lugares físicos adecuados como plazas, parques, playones, etc.

3) Si bien es cierto que es una zona de clubes de fútbol infantil, la gran mayoría de los chicos del barrio no tienen acceso al pago de las cuotas y, por otro lado, sus juegos están fuertemente condicionados por una sola práctica, la del fútbol. En este sentido creemos en la necesidad de ofrecer otras opciones y potenciar su bagaje lúdico, a partir de vivenciar otras experiencias de juego.

Los niños y jóvenes que viven en este contexto barrial, aparecen como el emergente de un largo proceso histórico-político que ha dejado profundas cicatrices en sus estructuras familiares, estos años de exclusión sistemática, produjeron agotadoras presiones sociales y económicas, que generaron una inigualable pauperización de la forma de vida de adultos, niñas y niños, empujando a todos a solventar la subsistencia de diversas maneras, ocupando para tal fin, gran parte del tiempo en tareas laborales.

Como consecuencia de lo anterior, una disgregación o directamente un abandono de las prácticas lúdicas, produjo una transformación de la cultura lúdica, modificando las posibilidades de interacción y socialización que el juego promueve. Estimamos que esto ha llevado, entre otras cosas, a potenciar mecanismos de individualización, atentando contra los vínculos comunales que el juego social que los contextos barriales permitieron en otros tiempos.

Por otra parte, el estado ha desatendido estas cuestiones y no ha podido insertar a través de sus diferentes redes de acción un espacio que garantice, no sólo la asistencia de bienes de consumo y planes de subsidios, que si bien para muchos pueden ser imprescindibles, sólo cubren una parte de la necesidad de los sujetos. En este sentido, no se tiene en cuenta que el jugar, "entendido como una forma genérica de la diversión" (Munné: 1980) es también un derecho no contemplado en las 
políticas estatales. Probablemente se piense que el juego aparece como consecuencia de un proceso natural en los sujetos, sin embargo, sostenemos que es una "construcción cultural" que debe ser garantizada, pues el jugar supone también un proceso de aprendizaje que permitirá la apropiación de un universo de sentidos y significados que sólo el medio y el contexto en el que se inserten podrá contenerlos. Asimismo, la educación popular no es más que la posibilidad de inclusión real y eficiente de sectores históricamente postergados, como sujetos activos de esa producción de saber, y aquí, la tarea por parte de los mediadores públicos, sean del estado, ONGs (organizaciones no gubernamentales), o los proyectos de extensión universitaria como en nuestro caso, son importantes, siempre que entendamos esta relación como una posibilidad de intercambio dialéctico.

\section{OBJETIVOS}

\section{Objetivo General:}

- Generar un espacio lúdico, que permita recuperar el jugar como práctica, derecho y necesidad.

- Profundizar en la extensión universitaria como práctica social y académica.

\section{Objetivos Específicos:}

- Lograr autonomía en la construcción de las prácticas lúdicas, con independencia de los facilitadores de los talleres.

- Recuperación de formas de juegos pasadas, a partir de la inserción comunitaria de juegos tradicionales.

- Posibilitar el reencuentro con la identidad originaria a partir de las maneras de jugar de los pueblos indígenas americanos.

- Fomentar la creatividad e invención, a partir del uso de materiales no convencionales para jugar.

- Desarrollar la formación e iniciación de los miembros del equipo en extensión.

\section{AlCANCES Y LIMITACIONES DE LA PROPUESTA}

Uno de los principales logros como extensionistas fue la posibilidad de confrontar aquellos saberes adquiridos en la formación con las nuevas situaciones de enseñanza y aprendizajes, surgidas tanto en las discusiones cotidianas como en las reuniones programadas (en torno a la planificación o las acciones concretas). Estas relaciones, comparaciones, críticas, idas y vueltas sobre lo que creíamos saber y aquello que observábamos, nos permitieron movernos del habitual lugar de poseedores de "conocimientos" (que iban al barrio a enseñar), para establecer nuevas relaciones de diálogo.

Nuestra posición había sido la de generar nuevos significados de nuestros saberes a partir de su puesta en práctica, y a la vez, tratar de que los niños y niñas que 
participan del espacio que proponemos puedan resignificar, en algunos casos, y significar, en otros, las prácticas y los saberes que circularon como propuestas lúdicas.

La práctica de extensión, en este tiempo y espacio que hemos propuesto, cobra un sentido diferente a la perspectiva tradicional de la extensión universitaria (al menos tal como la habíamos entendido), puesto que logramos poner en tensión nuestras percepciones y certezas para construir nuevos significados a partir de estas construcciones cotidianas en el patio de la escuela. De este modo, los objetivos del proyecto que apuntaban específicamente a la formación de los extensionistas fueron alcanzados.

Por otro lado, en función de los resultados esperados consignados en el proyecto, estamos en condiciones de afirmar que, por el momento, pudimos alcanzar en forma parcial los objetivos propuestos. Con respecto a uno de ellos: "Que los participantes aprendan a jugar, es decir: negociar, consensuar y acordar con los otros, la posibilidad de apropiarse del momento del juego, construyendo una cultura lúdica.", creemos que ha sido cumplido, en tanto y en cuanto el espacio permitió a los niños y niñas jugar de diversos modos, construyendo en ellos la posibilidad de poder ver a los compañeros de juego como partícipes necesarios de los mismos, y a partir de ello, poder negociar, consensuar y acordar para poder jugar. Es en ese sentido que podemos afirmar, al menos con el grupo de niños y niñas que asistieron con regularidad al espacio propuesto, que el objetivo fue alcanzado.

En el segundo ítem de los resultados esperados, consignábamos en el proyecto: "Que los participantes de los talleres logren jugar con cierta autonomía e independencia, logrando hacer extensivo sus momentos de juego por fuera de las propuestas del equipo", aparecen algunos indicadores que surgen a partir de algunos registros anecdóticos, que dan cuenta de como este resultado esperado surgió en ciertos momentos. Por ejemplo, luego de varios registros se puede leer reiteradas veces la siguiente referencia: "logran jugar con el material propuesto sin que aparezcan las consignas del docente" o bien "surge del relato de los niños/as que pudieron jugar en sus casas algunos de los juegos realizados en la escuela".

En el tercer ítem, "Que los niños, niñas y jóvenes tengan la oportunidad concreta de aprender nuevos juegos", es donde hemos tenido las mayores dificultades. Aquí concluimos que fue complejo lograr articular, durante el desarrollo del proyecto, los cuatro talleres originalmente propuestos. No obstante, pudimos ofrecer algunas variantes de juegos tradicionales o juegos con material alternativo. Sin embargo, tal y como lo habíamos previsto, este resultado esperado aún no ha sido alcanzado en su totalidad.

Los ítems restantes: "Formar alumnos y graduados como extensionistas" y "Observar y registrar las prácticas desarrolladas durante la experiencia, con el objeto de ser presentadas en encuentros de extensión y de divulgación académica", sostenemos fueron alcanzados.

El equipo ha realizado diversos informes y registros, sean estos individuales o colectivos, los mismos fueron utilizados como fuente para la elaboración del informe de avance, informe final, y trabajos de divulgación que han sido presentados en Congresos y Jornadas de divulgación académicas.

En líneas generales, consideramos que el objetivo "Generar un espacio lúdico, que permita recuperar el jugar como práctica, derecho y necesidad", lo hemos logrado 
parcialmente; logro que es sumamente significativo en términos cualitativos, pero que necesita de una mayor consolidación, de una progresión temporal; y ésto puede lograrse con políticas universitarias que sostengan estos proyectos a largo plazo.

Por último, consideramos importante destacar los efectos positivos no previstos en el proyecto inicial:

- Hay un grupo de niños y niñas que se identifican fuertemente con el proyecto y con el equipo de extensión, generando lazos afectivos que enriquecen las relaciones de los grupos. Concretamente, asisten sistemáticamente a la escuela a jugar.

- El equipo de extensión ha logrado un reconocimiento por parte de la institución escolar. Da cuenta de ello, que las directoras de ambas escuelas permiten a los extensionistas ingresar a las mismas en días diferentes a los que se desarrollan las tareas propias de extensión; los extensionistas hemos sido invitados a diversos eventos organizados por las escuelas, tales como festivales solidarios, actos de fin de curso, etc. Ante situaciones no previstas que afectan el funcionamiento de la escuela los días sábados, el equipo es consultado o avisado con anterioridad pudiendo prever las acciones a seguir, además de facilitarnos un espacio propio para guardar nuestros materiales.

En relación a la comparación a la situación inicial con la actual, creemos que ha habido ciertos avances. Una primera apreciación, tanto en términos empíricos inmediatos como a partir de la lectura de los registros, es que en el transcurso del tiempo, el grupo de niños y niñas que tuvieron continuidad en la participación de la propuesta, pudieron apropiarse de la misma. Muchos de ellos, pasaron de una posición de pasividad en términos de las prácticas lúdicas (en el sentido que esperaban que los extensionistas propusiéramos a qué y cómo jugar), a una actitud mucho más activa y de participación. Crearon juegos que los extensionistas no habíamos pensado, modificaron aquellos propuestos por nosotros, se apropiaron de todos los espacios de la escuela como no pueden hacerlo durante el ciclo regular de clases, creemos que por restricciones propias de la institución escolar, pero fundamentalmente porque el espacio escolar se presenta como un escenario diferente de apropiación. Para estos grupos, ir a jugar a la escuela los sábados a la mañana, se instaló como una práctica importante, una alternativa no prevista, en la que disfrutan, se divierten, intercambian, negocian, aprenden, juegan.

\section{A MODO DE CONCLUSIÓN}

Para finalizar este artículo proponemos algunas reflexiones surgidas a partir de los dos años y medio de trabajo, que dan cuenta de las acciones desarrolladas por el equipo en su conjunto.

a) Elaboramos estrategias de acomodamiento a las complejas dinámicas comunicacionales y de puesta en práctica de lo planificado, flexibilizando la propuesta de los talleres y proponiendo juegos sin orientaciones específicas. 
b) Constatamos las dificultades de llevar adelante una propuesta de carácter social, con anclaje en una realidad cambiante y cargada de imprevistos, permitiéndonos ser parte de una rica experiencia empírica y de replanteamientos teóricos. Aquí coincidimos en que suponer el juego como contenido educativo, lo ubica en una lógica que no es ni buena ni mala en sí misma, sino diferente. Nuestra pretensión de establecer prácticas lúdicas distintas a las propuestas escolares, respondía a cierta idealización teórica. Si bien el debate no está resuelto, estimamos establecer nuevas formas de abordaje en lo relacionado al juego, el aprendizaje, la enseñanza, la escolaridad y el tiempo libre.

Por otro lado, apareció un nuevo emergente empírico no contemplado: "el aburrimiento", situación que se observaba en algunos niños luego de experimentar los juegos motores propuestos por nosotros, pues los mismos agotaban rápidamente la actividad. En este caso eran juegos con cierta continuidad y correspondencia con juegos con acento en lo deportivo y por tanto con la lógica escolar. La pelota de básquetbol era utilizada en la cancha con los aros, las colchonetas tendían a las destrezas, la pelota de voleibol se utilizaba para jugar con una red por medio, usando cierta estructura del deporte. La propuesta entonces fue eliminar paulatinamente los elementos más convencionales, como así también modificar los espacios de juego, para que aparezcan en escena nuevos elementos, muchos de ellos desconocidos: latas, trompos, baleros, botellas plásticas, etc. La idea fue pensada en este sentido: la manipulación de elementos no habituales permitiría la posibilidad de nuevos acuerdos grupales en torno a los juegos, la elaboración de reglas, nuevas convenciones, etc., comprometería a los niños y niñas a hacerse cargo de sus propias decisiones.

Fue así que esta propuesta grupal que pensamos en torno a la reflexión de lo que ocurría, fue uno de los hallazgos más interesantes, puesto que las actividades de aquí en más, que propusieron los chicos y chicas, partían de sus propias necesidades, y eran vivenciadas de una manera muy particular, evidenciado en el compromiso asumido, pero fundamentalmente divertidas, lo cual mostraba la contracara del aburrimiento inicial.

c) Profundizamos lazos intercomunicacionales, accediendo al intercambio y debate a partir de las vivencias de cada uno de los extensionistas en el proyecto. Lo que nos hizo suponer que el camino recorrido hasta aquí nos posibilitó un proceso de aprendizaje.

Sin embargo, la puesta en marcha del proyecto, no ha tenido el impacto en la comunidad que hubiéramos deseado, por varios factores. Adelantamos algunos aunque sin certezas. En el contexto antes descrito, la escuela, como institución pública, no tiene una presencia destacada, dadas las particulares coyunturas políticas y sociales. Por lo tanto, un proyecto que se enmarca dentro de ese ámbito, pierde relevancia por no estar ajeno a las condiciones sociales que circulan como representación dentro del imaginario barrial. Si bien es importante destacar que, esta escuela en particular, desarrolla una actividad 
social sumamente importante, por lo tanto habrá elementos específicos que aún no podemos detectar.

Asimismo, podríamos inferir que: dada la cantidad de alumnos que asisten a las dos escuelas, sumado a ello los niños, niñas y jóvenes que circulan por el barrio, y teniendo en cuenta el número de participantes en el proyecto, no es cuantitativamente importante la asistencia por parte de los niños/as a "la escuela en juego". Aunque, más allá de los argumentos antes esgrimidos, sí tuvo un impacto altamente positivo en el grupo que participó sistemáticamente en la propuesta y en la comunidad educativa que conforman las dos instituciones escolares. Del grupo que asistió originalmente a "La escuela en juego" en el año 2007 hoy participan veinte alumnos, los que pudieron comprender y transmitir la lógica lúdica de la flexibilidad de la regla, del juego compartido, cooperación y compromiso al resto de los nuevos "jugadores" que aún hoy se siguen sumando a la propuesta. En cuanto a las posibilidades intracomunicacionales entre las dos instituciones que apoyaron las actividades se lograron potenciar acuerdos de colaboración mutua a partir de lo que significó compartir un mismo espacio de práctica, un día no habitual de clases. La sola explicación del marco teórico que la fundamenta, como así también la visibilidad de la práctica, permitió que los educadores que no estaban dentro del proyecto se preguntaran acerca del rol del juego en la lógica escolar. Eso que para nosotros era una preocupación teórico-práctica terminó siéndolo -al menos en parte- una curiosidad para otros. Creemos que instalar un debate también es un logro de todos los que hacemos la extensión.

\section{REFERENCIAS BibLIOGRÁfiCAS}

AUGÉ, M. (2000). Los no lugares. Espacios del anonimato. Barcelona: Gedisa.

BUSTELO, E. (2007). El recreo de la infancia. Argumentos para otro comienzo. Buenos Aires: Siglo XXI Editores.

EGUÍA, A.; GILES, M.; ORTALE, S.; PEIRO, L.; RAUSKY, E. Y VIÑES, N. (2008). "Reflexiones, Debates, Consensos y Discrepancias. Experiencias de los Proyectos de Extensión de la FaHCE. Facultad de Humanidades y Ciencias de la Educación, Universidad Nacional de La Plata". (Inédito).

MUNNÉ, F. (1980). Psicosociología del tiempo libre. Un enfoque crítico. México: Trillaso.

SARLÉ, M. (2006). Enseñar el juego y jugar la enseñanza. Buenos Aires: Paidos.

Secretaría de Extensión Universitaria, Universidad Nacional de La Plata (2007). "Bases para la convocatoria de proyectos de extensión universitaria". 